\title{
NUMBER DENSITY PREDICTIONS FOR PRIMEVAL GALAXIES
}

\author{
E.THOMMES \& K.MEISENHEIMER \\ Max-Planck-Institut für Astronomie, \\ Königstuhl 17, D-69117 Heidelberg, Germany
}

In the last two decades several methods have been employed to search for the ancestors of present day galaxies undergoing their first starburst at high redshift, the so-called ,primeval galaxies" (PGs). According to predictions of the Ly- $\alpha$ flux from the PG phase current surveys for Ly- $\alpha$ bright PGs should have detected $10^{1}-10^{3}$ PGs. Yet, no really good candidate has been found. One possible explanation for this discrepancy would be the presence of dust which could strongly suppress Ly- $\alpha$. In fact, models of early galaxy formation are able to produce gas to dust ratios of about $1 / 10$ solar after some $10^{8}$ years. This would suppress the emerging Ly- $\alpha$ flux below current detection limits. Thus, to detect a PG in its Ly- $\alpha$ bright phase, one has to hit exactly the redshift of their first star formation. On the other hand, the epoch of galaxy formation is likely to extend over much longer time. So when searching a finite volume of the universe (given by the depth $\Delta z$ and the field $\Delta \Omega$ ), the protogalaxies are not simultaniously in the Ly- $\alpha$ bright PG phase and only a fraction of them exceed the detection limit. Our new attempt to predict the expected number of PGs takes this reduced propability into account. Further contraints of the Ly- $\alpha$ luminosity were derived from recent observations and theoretical work (for details see E.Thommes \& K.Meisenheimer in Galaxies in the Young Universe, Eds. H.Hippelein et al., Springer-Verlag, 1995, p.242). As expected, we find that the number density of Ly- $\alpha$ emitting PGs is drastically reduced in comparison to previous predictions. Thus previous surveys had no realistic chance to find them. Nevertheless, our calculations also show, that the detection of Ly- $\alpha$ bright PGs is in the scope of present day techniques. Specifically, we predict that the Calar-Alto-Deep-Imaging-Survey (see contribution of H. Hippelein et. al., these proc.) which will search for Ly- $\alpha$ in an area of $\approx 0.3 \square^{\circ}$ down to a limiting line flux of $3 \times 10^{-20} \mathrm{~W} / \mathrm{m}^{2}$ should detect 10 $\ldots 100 \mathrm{PGs}$ in three redshift intervals $\Delta z=0.1$. The uncertainty accounts for our ignorance of $q_{0}$ and the unknown epoch of galaxy formation. 